\title{
Indicadores antropométricos e risco para doenças cardiovasculares em mulheres com doenças reumáticas ${ }^{1}$
}

\section{Anthropometric indicators and risk for cardiovascular diseases in women with rheumatic diseases}

\author{
Eloá Angélica Koehnlein²; Rose Mari Bennemann³
}

\section{Resumo}

O objetivo deste trabalho foi avaliar o estado nutricional e o risco para doenças cardiovasculares de mulheres com doenças reumáticas em uma associação de apoio ao reumático de Maringá-PR. $\mathrm{O}$ estado nutricional foi determinado por meio do Índice de Massa Corporal (IMC) e o risco para doenças cardiovasculares a partir da razão cintura-quadril (RCQ) e da circunferência da cintura isolada (CC). Foram avaliadas 43 mulheres, sendo 11 adultas e 32 idosas. A maioria $(60,46 \%)$ das mulheres avaliadas apresentou excesso de peso, 32,56\% apresentaram peso adequado e 6,98\% (3 idosas) apresentaram baixo peso. O risco para doenças cardiovasculares foi observado em $48,84 \%$ das mulheres, de acordo com a avaliação pela RCQ. Já a avaliação da CC isolada demonstrou que $83,72 \%$ das mulheres apresentavam risco. Conclui-se que a prevalência de excesso de peso e de risco para doenças cardiovasculares é preocupante nesta população, não apenas em decorrência das complicações associadas, mas pelo agravamento ainda maior na qualidade de vida desses indivíduos.

Palavras-chave: Doenças reumáticas. Índice de massa corporal. Razão cintura-quadril.Circunferência da cintura. Risco para doenças cardiovasculares.

\begin{abstract}
The purpose of this work was to evaluate the nutritional status and the risk for cardiovascular diseases in women with rheumatic diseases from an association for patients with rheumatic diseases in Maringá - PR. The nutritional status was determined by means of the Body Mass Index (BMI) and the risk for cardiovascular diseases based on the waist-to-hip ratio (WHR) and the isolated waist circumference (WC). Forty-three women were evaluated, being 11 adults and 32 elderly. Most of the evaluated women (60.46\%) showed to be overweight, $32.56 \%$ had appropriate body weight and $6.98 \%$ had low weight. The risk for cardiovascular diseases was observed in $48.84 \%$ of the women, according to the waist-tohip evaluation. The isolated WC showed that $83.72 \%$ of the women had risk of cardiovascular diseases. It is concluded that the prevalence of overweight and risk for cardiovascular diseases is worrying for this population, not only because of the associated complications, but also for the worsening in the life quality of these individuals.
\end{abstract}

Key words: Rheumatic diseases. Body Mass Index. Waist-to-hip ratio. Waist circumference. Risk for cardiovascular diseases.

\footnotetext{
1 Projeto de Iniciação Científica (PICC) do Centro Universitário de Maringá-CESUMAR 2006/2007. Maringá-PR

2 Nutricionista pelo Centro Universitário de Maringá-PR. E-mail: eloa-angelica@hotmail.com

3 Nutricionista, docente do curso de nutrição do Centro Universitário de Maringá-PR. Mestre e doutoranda em Saúde Pública pela Faculdade de Saúde Pública (FSP) da Universidade de São Paulo (USP). E-mail: rosemari@cesumar.br
} 


\section{Introdução}

Em decorrência do envelhecimento mundial da população, as doenças reumáticas vêm apresentando incidência crescente. Essa família de doenças inclui uma série de manifestações no tecido conjuntivo afetando freqüentemente os vasos sanguíneos, os tecidos intersticiais, cartilagens, ossos, tendões, ligamentos e membranas sinoviais (FRANZESE, 2002).

Sob a denominação de doenças reumáticas estão incluídas inúmeras doenças, que segundo a Sociedade Brasileira de Reumatologia (2006), são divididas didaticamente nestas categorias: doenças difusas do tecido conjuntivo, como o lúpus eritematoso sistêmico e a artrite reumatóide; vasculites sistêmicas; espondiloartropatias, como a espondilite anquilosante; doenças osteometabólicas, como a osteoporose e a osteomalácea; doenças articulares degenerativas, como a osteoartrose; artropatias microcristalinas, como a gota; artropatias reativas, como a osteomielite e a febre reumática; reumatismos extra-articulares, como a fibromialgia, tendinites e bursites; artrites intermitentes, como a hidrartrose; e artropatias secundárias a outras doenças não-reumáticas.

Todas essas doenças, apesar de apresentarem etiologia desconhecida, estão relacionadas a fatores genéticos, hormonais e ambientais (SOCIEDADE BRASILEIRA DE REUMATOLOGIA, 2006). Zuber (1997) atribui a etiologia patognomônica a um estresse contínuo, como o excesso de peso, o exercício extenuante ou inadequado, a infecção viral e, ainda, a resposta auto-imune, que leva a um processo inflamatório contínuo.

Essa família de doenças conduz a incapacidade do paciente, o que reflete na economia do país, pela redução da produtividade, gastos com assistência médica/hospitalar e auxílio-doença, e, ainda, representa um sério risco de saúde. No entanto, pouca atenção é dedicada ao papel do estado nutricional e da dieta em sua progressão e gravidade (FRANZESE, 2002; GALPERIN; GERMAN; GERSHWIN, 2003).
As doenças reumáticas e inflamatórias podem levar a distúrbios nutricionais, em virtude da alta produção de citocinas, aumento da taxa metabólica, limitação na realização das atividades de vida diária (AVDs) e complicações gastrintestinais resultantes da própria doença e dos medicamentos utilizados principalmente à desnutrição, que contribui para aumento da morbi-mortalidade desses pacientes. A desnutrição predispõe o paciente a uma série de complicações adicionais como tendência a infecção, deficiência na cicatrização, falência respiratória, insuficiência cardíaca, diminuição da síntese de proteínas, diminuição da filtração glomerular e da produção de suco gástrico (ACUNÃ; CRUZ, 2004; GALPERIN; GERMAN; GERSHWIN, 2003).

As alterações provocadas no trato gastrintestinal, embora dependam do tipo de doença difusa do tecido conjuntivo, podem conduzir à desnutrição, uma vez que interferem na ingestão e absorção dos nutrientes (MACHADO et al., 2004). Dentre essas alterações, pode-se citar: anormalidades da cavidade oral, como limitação na abertura da boca (por alteração da articulação temporomandibular), dor ao mastigar e dificuldade de deglutição, seja por complicações da própria doença reumática ou como conseqüência da Síndrome de Sjogren; disfunção esofagiana com disfagia ou odinofagia, má absorção e enteropatia perdedora de proteínas (GALPERIN; GERMAN; GERSHEIN, 2003).

Alémdascomplicações resultantesdadesnutrição, a obesidade também é um fator complicador do quadro de doenças como a osteoartrite e a artrite reumatóide, uma vez que sobrecarrega as articulações já lesionadas. Ela ainda está associada, no caso da gota, à redução da excreção de uratos, bem como predispõe mais freqüentemente a doença isquêmica do coração, hipertensão arterial, acidente vascular cerebral, diabetes melito tipo 2, colelítiase, neoplasias e esofagite de refluxo (RADOMINSKI, 1998; ACUNÃ; CRUZ, 2004). Essa condição pode ser resultado da limitação na prática das AVDs e de atividade física imposta pela doença (FRANZESE, 2002). 
A preocupação com o estado nutricional decorre também dos distúrbios metabólicos decorrentes do uso de antiinflamatórios não-esteroidais comuns nestas patologias e, ainda, em virtude do aumento da taxa metabólica secundária ao processo de inflamação (ESCOTT-STUMP, 1999).

Os distúrbios nutricionais ganham maior grau de complexidade quando o paciente é idoso, uma vez que este já está sujeito às alterações inerentes ao próprio processo de envelhecimento e à polimedicação, que influenciam na capacidade funcional, na ingestão dos alimentos, na digestão e absorção dos nutrientes, bem como nos processos metabólicos (CAMPOS; MONTEIRO; ORNELAS, 2000).

Dessa forma, este trabalho teve como objetivo avaliar o estado nutricional e o risco para doenças cardiovasculares em mulheres adultas e idosas com doenças reumáticas.

\section{Material e métodos}

O presente estudo foi descritivo, transversal e realizado com a coleta de dados primários.

Foram convidados a participar da pesquisa indivíduos com idade superior a 20 anos, freqüentadores regulares de uma associação de apoio ao reumático de Maringá-PR. Em função da maioria de os freqüentadores serem do sexo feminino, optou-se por estudar somente esta população. Foi solicitada a autorização da associação, bem como a assinatura do termo de consentimento livre e esclarecido a todos os indivíduos que aceitaram participar da pesquisa. O projeto foi aprovado pelo Comitê Permanente de Ética em Pesquisa do Centro Universitário de Maringá.

Para participar da associação, o indivíduo deve apresentar atestado médico comprovando possuir doença reumática. Dessa forma, todas as avaliadas possuíam doença reumática. No entanto, não foi especificado o tipo de doença reumática, pois, além do acesso aos atestados não ter sido permitido, as mulheres também não souberam informá-lo.
O estado nutricional foi avaliado a partir do Índice de Massa Corporal (IMC) e o risco para doenças cardiovasculares por meio da razão cintura-quadril (RCQ) e da circunferência da cintura (CC) isolada. As medidas coletadas no estudo foram: peso, estatura, circunferência da cintura e do quadril.

O peso foi medido por meio de uma balança digital portátil, marca Plenna ${ }^{\circledR}$, com capacidade para $150 \mathrm{Kg}$ e sensibilidade de $100 \mathrm{~g}$. As mulheres foram pesadas em pé, vestindo roupas leves e sem sapatos. A estatura foi medida utilizando fita antropométrica inextensível, com variação em milímetros, fixada a parede desprovida de rodapé. As circunferências da cintura (CC) e do quadril (CQ) foram medidas com fita antropométrica inextensível, com variação em milímetros. A CC foi medida na menor curvatura observada entre a última costela e a crista ilíaca e a CQ foi medida na maior protuberância observada entre a cintura e as coxas. As medidas de peso e estatura foram realizadas segundo a técnica de Gordon, Chumlea e Roche (1988) e a CC e CQ segundo a técnica de Callway et al. (1988).

O Índice de Massa Corporal (IMC) foi calculado dividindo-se o peso corporal $(\mathrm{kg})$, pela estatura $(\mathrm{m})$ elevada ao quadrado $\left(\mathrm{P} / \mathrm{E}^{2}\right)$. A razão cintura/quadril foi obtida por meio da divisão da medida da CC pela CQ (CC/CQ).

O estado nutricional das mulheres adultas $(\geq 20 \leq$ 59 anos) foi classificado de acordo com os pontos de corte preconizados pela World Health Organizaton (WHO, 1997). Para as mulheres idosas $(\geq 60$ anos) (BRASIL, 2003) foi utilizada a classificação proposta pelo Nutrition Screening Initiative (1992) que considera os valores de IMC entre $22-27 \mathrm{Kg} / \mathrm{m}^{2}$ como peso adequado. No presente estudo, além da classificação proposta pelo NSI, considerou-se com baixo peso as mulheres idosas que apresentaram índices $<22 \mathrm{Kg} / \mathrm{m}^{2}$ e com excesso de peso as que apresentaram índices $>27 \mathrm{Kg} / \mathrm{m}^{2}$.

$\mathrm{O}$ risco para doenças cardiovasculares (DCVs), tanto para as mulheres adultas quanto para as idosas, foi determinado por meio da razão cintura/quadril e 
da circunferência da cintura, utilizando-se os pontos de corte preconizados pela WHO (1997).

Os dados coletados foram analisados por meio de estatística descritiva. Para as variáveis qualitativas, foram utilizadas as freqüências absolutas (n) e as freqüências relativas (\%) e para as variáveis quantitativas, os resultados foram apresentados em médias, desvios-padrão e medianas. Os cálculos foram realizados por meio do programa Statistica 6.0 .

\section{Resultados e discussão}

A população estudada foi composta por 43 mulheres, sendo 11 adultas e 32 idosas. A idade média da população foi de $64,35 \pm 9,62$ anos, sendo $68,87 \pm 5,10$ para as mulheres idosas e $50,9 \pm 7,58$ para as mulheres adultas. As médias, medianas e desvios-padrão das variáveis estudadas podem ser observadas na Tabela 1 .

Tabela 1. Médias, desvios-padrão e medianas das variáveis estudadas em mulheres com doenças reumáticas em Maringá-PR.

\begin{tabular}{cccc}
\hline Variável & média & \pm desvio-padrão & mediana \\
\hline Idade (anos) & 64,36 & 9,62 & 65,5 \\
Peso $(\mathrm{Kg})$ & 68,96 & 13,35 & 65,15 \\
Estatura (m) & 1,56 & 0,07 & 1,56 \\
IMC (Kg/m $\left.{ }^{2}\right)$ & 28,37 & 5,81 & 27,78 \\
CC (cm) & 90,58 & 11,02 & 90,25 \\
CQ (cm) & 105,17 & 10,80 & 102,75 \\
RCQ & 0,86 & 0,08 & 0,85 \\
\hline
\end{tabular}

A maior prevalência de doenças reumáticas em mulheres pode ser observada no Programa contra as doenças reumáticas de Portugal (2004), que apontou como público-alvo para as ações mulheres na pós-menopausa e idosos. Segundo Fellet et al. (2002), para cada homem, há 3 a 4 mulheres com artrite reumatóide e 9 mulheres para cada homem com lúpus eritematoso sistêmico. Esta tendência só não é observada em relação à gota.

A maior prevalência do sexo feminino, sobretudo de mulheres maduras no final da idade fértil, pode ser explicada pelo caráter auto-imune das doenças reumáticas, uma vez que a auto-imunidade parece ter relação com os hormônios sexuais (BARCELLOS; ANDRADE, 2004).
Dada a escassez de trabalhos a respeito de avaliação nutricional e risco para doenças cardiovasculares em indivíduos com doenças reumáticas, cabe esclarecer que os dados antropométricos foram comparados com publicações que avaliaram indivíduos adultos e idosos com ou sem esse tipo de doença.

Observou-se IMC médio de 25,93 $\pm 3,79 \mathrm{Kg} /$ $\mathrm{m}^{2}$ para as mulheres adultas e $29,03 \pm 6,24 \mathrm{Kg} / \mathrm{m}^{2}$ para as idosas. Sampaio e Figueiredo (2005), ao estudarem indivíduos adultos e idosos, encontraram na população feminina adulta IMC médio de 27,10 $\mathrm{Kg} / \mathrm{m}^{2}$ e na população idosa IMC médio de 27,30 $\mathrm{Kg} / \mathrm{m}^{2}$. 
A avaliação do estado nutricional por meio do IMC mostrou que o excesso de peso predominou na população avaliada. O baixo peso foi encontrado somente no grupo das mulheres idosas $(9,37 \%)$ (Figura 1).

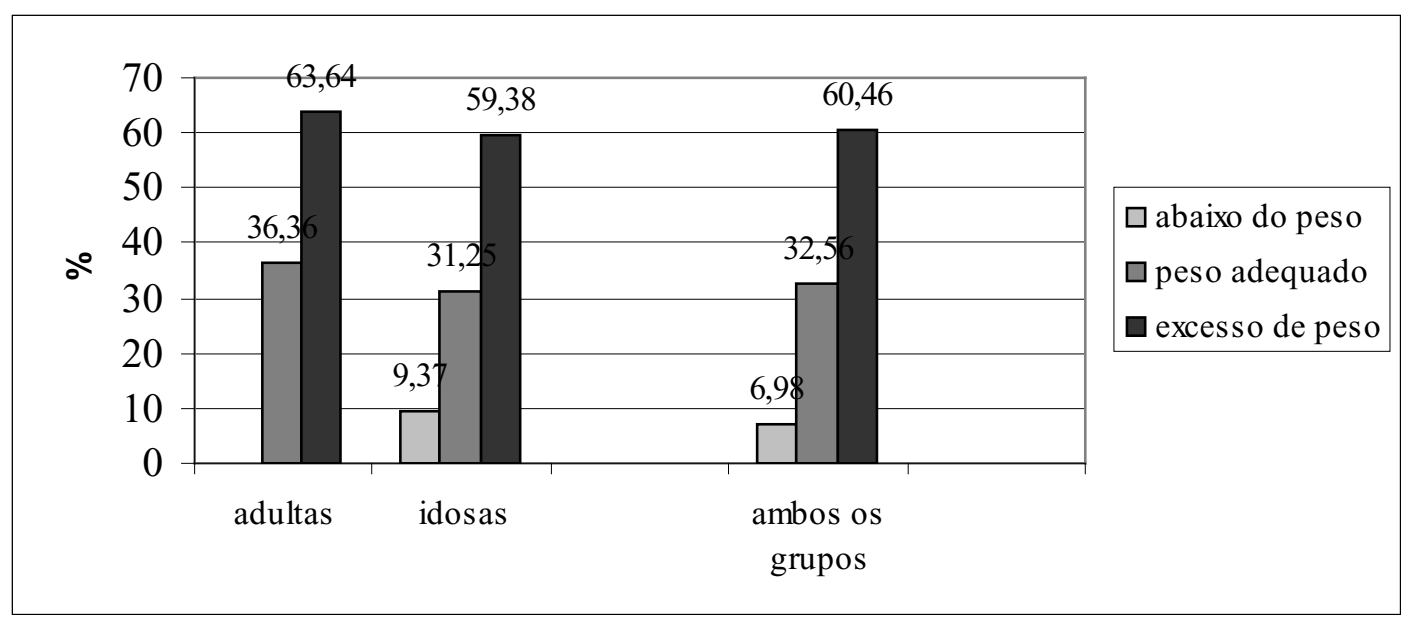

Figura 1. Estado nutricional das mulheres com doenças reumáticas, pelo IMC, segundo grupo etário.

Esses resultados configuram o oposto do que era esperado (baixo peso), uma vez que as doenças reumáticas, por fatores determinados pela própria doença e pelos medicamentos utilizados, conduzem a anormalidades nos processos de ingestão, digestão e absorção dos nutrientes, bem como provocam um aumento na taxa metabólica pelo processo inflamatório, que conduziriam à desnutrição (FRANZESE, 2002).

Na população idosa, além das doenças reumáticas interferindo no estado nutricional, existem os fatores relacionados ao próprio processo de envelhecimento, que predispõem ao surgimento de distúrbios nutricionais, principalmente à desnutrição. Neste estudo, entretanto, a prevalência de excesso de peso e peso adequado foi maior nas mulheres idosas, já que $59,37 \%$ apresentaram excesso de peso e $31,25 \%$ peso adequado.

Cabrera et al. (2005), ao estudarem um grupo de mulheres idosas, observaram percentuais similares a este estudo para o excesso de peso e peso adequado. $\mathrm{O}$ excesso de peso foi observado em $55,80 \%$ das mulheres idosas e o peso adequado em $39,50 \%$.
Para o baixo peso, os resultados foram diferentes, e os pesquisadores encontraram $4,70 \%$ das avaliadas com este diagnóstico, enquanto este estudo verificou $9,37 \%$.

Como verificado no grupo das mulheres idosas, no grupo das adultas prevaleceu o excesso de peso $(63,64 \%)$ (Figura 1). Fernandes et al. (2005) também encontraram, ao estudar mulheres adultas, percentual elevado $(59,90 \%)$ com excesso de peso. De modo semelhante, Teichmann et al. (2006) encontraram, em São Leopoldo-RS, 59,70\% das mulheres adultas avaliadas com excesso de peso.

Percentuais diferentes aos deste estudo foram encontrados por Afonso e Sichieri (2002) ao estudarem mulheres adultas no Rio de Janeiro. O excesso de peso foi observado em $39,40 \%$ das mulheres adultas e o peso adequado em $65,80 \%$.

$\mathrm{O}$ alto percentual de mulheres com excesso de peso neste estudo é preocupante, uma vez que essa condição é um complicador adicional nas doenças reumáticas. Segundo Huang et al. (2000), que estudaram os efeitos da redução de peso em pacientes com osteoartrite, a perda de peso é um 
fator coadjuvante em sua reabilitação. Felson et al. (2004) também observaram que existe uma forte associação entre IMC elevado e progressão das doenças articulares. Shapiro, Anderson e DanoffBurg (2005), ao estudarem a relação entre IMC e fibromialgia, concluíram que a perda de peso deve fazer parte do tratamento de mulheres obesas com essa doença. Da mesma forma, Yunus, Arslan e Aldag (2002) concluíram, também com relação à fibromialgia, que a perda de peso pode melhorar a condição física dos pacientes.

Os altos índices de excesso de peso encontrados no presente estudo, provavelmente, devem-se às limitações físicas decorrentes dessas doenças, ao uso de antiinflamatórios não-hormonais, bem como a hábitos alimentares inadequados, atualmente observados, em todas as idades e nas mais diversas populações (GALPERIN; GERMAN; GERSHEWIN, 2003; INSTITUTO BRASILEIRO DE GEOGRAFIA E ESTATÍSTICA, 2004).

Nas mulheres adultas, a média da $\mathrm{CC}$ foi de $83,75 \pm 7,85 \mathrm{~cm}$ e nas idosas $92,52 \pm 11,21 \mathrm{~cm}$. Observa-se que o valor da média da CC das mulheres idosas é superior a das mulheres adultas. Esse resultado condiz com as informações disponíveis na literatura, de que, com o envelhecimento, o tecido adiposo subcutâneo aumenta no tronco e diminui nos membros (JACOB FILHO; SOUZA, 1994; WHO, 1995).

Cabe esclarecer que, como referência para CC, os valores de WHO (1997) também foram adotadas para as mulheres idosas, porque além de não existir uma referência estabelecida para analisar o risco para DCVs pela CC, específica para os idosos, a maioria dos estudos utiliza os pontos de corte preconizados pela WHO (1997). No Brasil, Barbosa et al. (2005) publicaram valores referentes a idosos da cidade de São Paulo, porém, não apresentaram pontos de corte que definissem risco para DCVs. No entanto, se forem utilizados como referência os valores apresentados por esses autores, considerandose como risco valores acima do percentil 75 , nas mulheres idosas, o risco para DCVs deixa de ser observado. Esse resultado pode ser explicado pelo fato da população do estudo referido ter sido constituída apenas de idosos, diferentemente da população considerada (indivíduos adultos) pela WHO (1997) para estabelecer os pontos de corte para a CC.

As médias da RCQ encontradas $0,81 \pm 0,05$ para as mulheres adultas e $0,88 \pm 0,08$ para as mulheres idosas foram superiores ao recomendado, ou seja, $\mathrm{RCQ}<0,8$, indicando risco para DCVs tanto para as mulheres adultas quanto para as idosas. Sampaio e Figueiredo (2005), ao avaliarem o risco para DCVs pela RCQ, encontraram risco para DCVs apenas nas mulheres idosas.

De modo semelhante à análise da $\mathrm{CC}$, a RCQ também não indicou risco para doenças cardiovasculares para as idosas, quando se realizou análise de acordo com Barbosa et al. (2005), utilizando como ponto de corte de risco para doenças cardiovasculares valores acima do percentil 75 .

Em relação ao risco cardiovascular estimado por meio da RCQ e CC isolada, observou-se que esta última apontou que mais de $80 \%$ das pacientes pesquisadas apresentavam risco, enquanto a primeira apontou esse risco para cerca de metade do grupo estudado. A análise dos grupos etários, separadamente, apontou que nas idosas, o risco para doenças cardiovasculares estimado tanto pela RCQ quanto pela CC isolada foi maior em comparação com as mulheres adultas. A distribuição percentual das mulheres, de acordo com a classificação da RCQ e da CC, pode ser observada nas figuras 2 e 3 , respectivamente. 


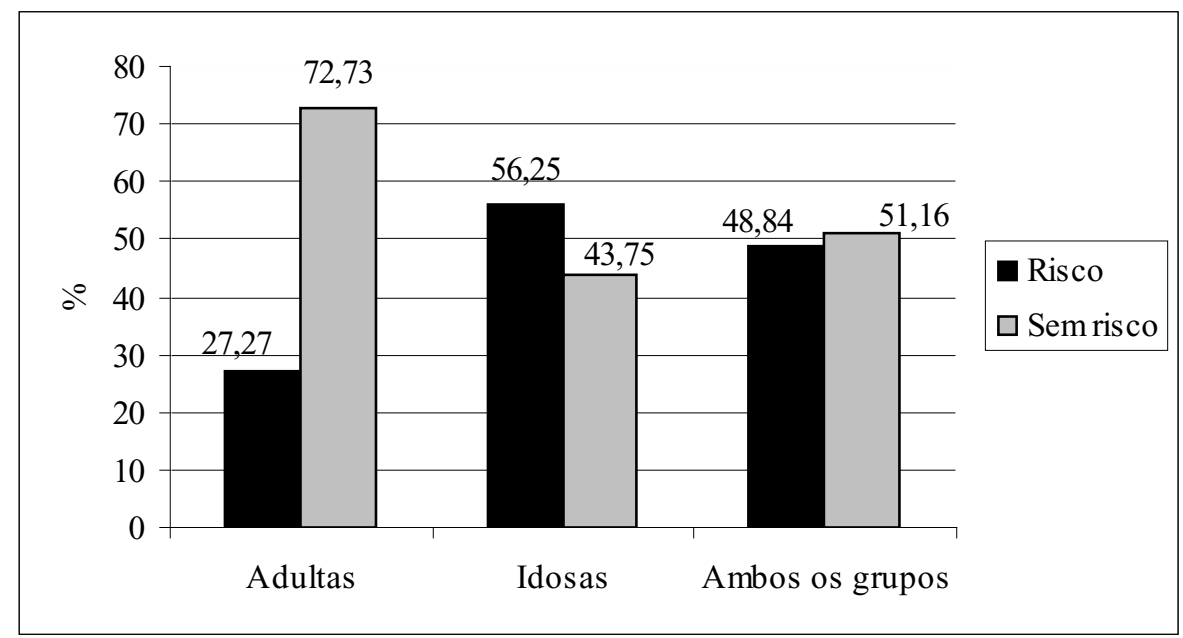

Figura 2. Presença de risco cardiovascular nas mulheres com doenças reumáticas, pela RCQ, de acordo com o grupo etário.

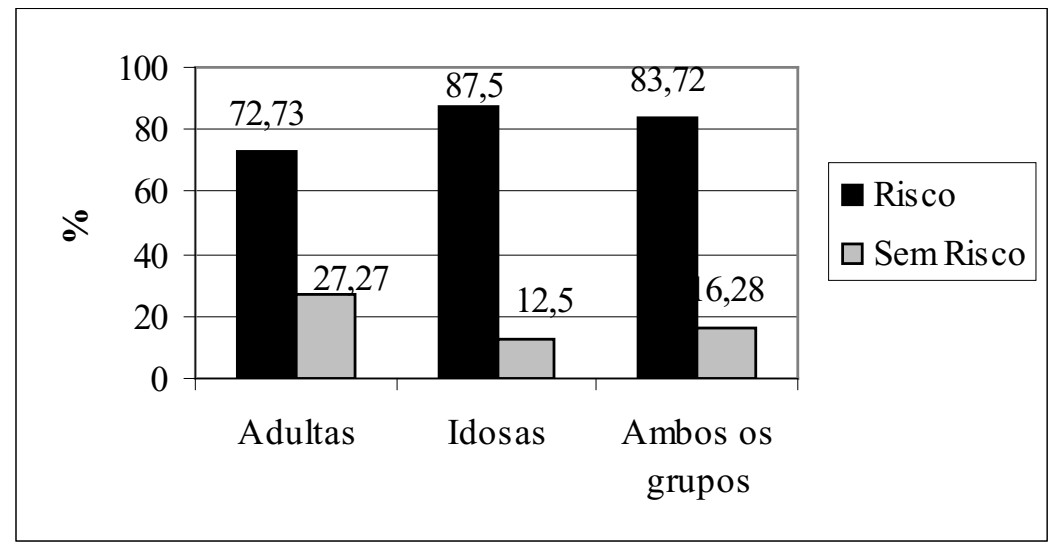

Figura 3. Presença de risco cardiovascular nas mulheres com doenças reumáticas, pela $\mathrm{CC}$, de acordo com o grupo etário.

Machado e Sichieri (2002) encontraram prevalência de 51,90\% de risco para doenças cardiovasculares em mulheres adultas, de acordo com a classificação da RCQ, enquanto este estudo encontrou $27,27 \%$. No entanto, para a população idosa, resultados semelhantes aos encontrados podem ser observados no estudo de Santos e Sichieri (2005), que verificou que $54,10 \%$ das idosas avaliadas apresentavam risco para doenças cardiovasculares, de acordo com a RCQ. Já em relação à $\mathrm{CC}$, índices menores aos deste estudo foram encontrados pelos mesmos autores, sendo e $65,9 \%$ das idosas apresentavam risco.
Na literatura, não se observa consenso em relação ao melhor preditor de risco cardiovascular. De acordo com Cabrera et al. (2005), nos idosos a RCQ reflete melhor a composição de gordura corpórea e seu papel deletério sobre a morbimortalidade. Sampaio (2004) e Olinto et al. (2006), por sua vez, já apontam que a CC parece correlacionar-se mais adequadamente com a gordura localizada em nível abdominal, sendo mais sensível que a RCQ para indicar risco cardiovascular, pois apresenta estreitas relações com alterações metabólicas. Segundo os autores, a RCQ tem demonstrado associação moderada com o acúmulo abdominal de gordura. 


\section{Conclusão}

Por meio deste estudo, foi possível observar que diferentemente do que se esperava para a população em questão, o excesso de peso prevaleceu sobre o baixo peso, atingindo mais da metade da população avaliada.

O risco para doenças cardiovasculares estimado através da CC e RCQ foi encontrado em ambos os grupos estudados (adultas e idosas), porém com maior freqüência na população idosa.

Cabe ressaltar as limitações dos resultados obtidos, em função do delineamento e tamanho da amostra e, portanto as conclusões não podem ser extrapoladas para todos os pacientes com doenças reumáticas.

Dessa forma, salienta-se a necessidade do desenvolvimento de mais pesquisas, com amostras mais abrangentes, sobre o estado nutricional dos indivíduos com doenças reumáticas, a fim de que se evite o aumento da morbimortalidade em decorrência de distúrbios nutricionais. A intervenção nutricional precoce, no sentido de reverter desvios como o excesso ou o baixo peso, pode prevenir o agravamento da doença reumática, não só evitando sua progressão, como também melhorando a qualidade de vida desses indivíduos.

\section{Referências}

ACUÑA, K.; CRUZ, T. Avaliação do estado nutricional de adultos e idosos e situação nutricional da população brasileira. Arquivos Brasileiros de Endocrinologia \& Metabologia, São Paulo, v. 48, n. 3, p. 345-361, jun. 2004.

AFONSO, F. M.; SICHIERI, R. Associação do índice de massa corporal e da relação cintura/quadril com hospitalizações em adultos do município do Rio de Janeiro, RJ. Revista Brasileira de Epidemiologia, São Paulo, v. 5, n. 2, p. 153-163, 2002.

BARBOSA, A. R.; SOUZA, J. M. P.; LEBRÃO, M. L.; LAURENTI, R.; MARUCCI, M. F. N. Anthropometry of elderly residents in the city of São Paulo, Brazil. Cadernos de Saúde Pública, Rio de Janeiro, v. 21, n. 6, p. 1929-1938, nov./dez. 2005.
BARCELLOS, K. S. A.; ANDRADE, L. E. C. Microquimerismo Fetal-Materno nas Doenças Reumáticas Auto-Imunes. Revista Brasileira de Reumatologia, São Paulo, v. 44, n. 1, p. 53-61, jan./fev. 2004.

BRASIL. Ministério da Saúde. Lei $n$. 10.741, de $1^{\circ}$ de outubro de 2003. Dispõe sobre o estatuto do idoso e dá outras providências. Legislação de Saúde. Brasília: Ministério da Saúde, 2003.

CABRERA, M. A. S.; WANJGARTEN, M.; GEBARA, O. C. E.; DIAMENT, J. Relação do índice de massa corporal, da relação cintura-quadril e da circunferência abdominal com a mortalidade em mulheres idosas: seguimento de 5 anos. Cadernos de Saúde Pública, Rio de Janeiro, v. 21, n. 3, p. 767-775, maio/jun. 2005.

CALLWAY, W. C.; CHUMLEA, W. C.; BOUCHARD, C.; HIRNES, J. H.; LOHMAN, T. G.; MARTIN, A. D.; MITCHELL, C. D.; MULLER, W. H.; ROCHE, A. F.; SEEFELDT, V. D. Circumferences. In: LOHMAN, T. G.; ROCHE, A. F.; MARTORELL R. Anthropometric standardization reference manual. Champaign, Illinois: Human Kinetics, 1988. p. 39-54.

CAMPOS, M. T. F. S.; MONTEIRO, J. B. R.; ORNELAS, A. P. R. C. Fatores que afetam o consumo alimentar e a nutrição do idoso. Revista de Nutrição, Campinas, v. 13, n. 3, p. 157-165, set./dez. 2000.

ESCOTT-STUMP, S. Nutrição relacionada ao diagnóstico e tratamento. 4. ed. São Paulo: Manole, 1999.

FELLET, A. J.; SCOTTON, A. S.; OLIVEIRA, W. M. L.; SCOTTON, A. C. B. S.; FRAGA, R. O. Artrite na mulher. Revista Brasileira de Medicina no Esporte, São Paulo, v. 59, n. 5, p. 307-317, maio 2002.

FELSON, D. T.; GOGGINS, J.; NIU, J.; ZHANG, Y.; HUNTER, D. J. The effect of body weight on progression of knee osteoarthritis is dependent on alignment. Arthritis \& Rheumatism, New York, v. 50, n. 12, p. 3904-3909, dez. 2004.

FERNANDES, A. M. S.; PAES LEME, L. C.; YAMADA, E. M.; SOLLERO, C. A. Avaliação do índice de massa corpórea em mulheres atendidas em ambulatório geral de ginecologia. Revista Brasileira de Ginecologia e Obstetrícia, Rio de Janeiro, v. 27, n. 2, p. 69-74, 2005.

FRANZESE, T. A. Terapia Clínica Nutricional nos Distúrbios Reumáticos. In: MAHAN, L. K.; ESCOTTSTUMP, S. Krause: alimentos, nutrição e dietoterapia. 10. ed. São Paulo: Roca, 2002. p. 937-951.

GALPERIN, C.; GERMAN, B. J.; GERSHWIN, M. E. Nutrição e dieta nas doenças reumáticas. In: SHILS, M. E.; OLSON. J. A.; SHIKE, M.; ROSS, A. C. Tratado 
de nutrição moderna na saúde e na doença. Barueri: Manole, 2003. p. 1417-1444.

GORDON, C. C.; CHUMLEA, W. C.; ROCHE, A. F. Stature, recumbent length and weight. In: LOHMAN, T. G.; ROCHE, A. F.; MARTORELL, R. Anthropometric standardization reference manual. Champaign, Illinois: Human Kinetics, 1988. p. 3-8.

HUANG, M. H.; CHEN, C. H.; CHEN, T. W.; WENG, M. C.; WANG, W. T.; WANG, Y. L. The effects of weight reduction on the rehabilitation of patients with knee osteoarthritis and obesity. Arthritis Care \& Research, New York, v. 13, n. 6, p. 398-405, dez. 2000.

JACOB FILHO, W.; SOUZA, R. R. Anatomia e fisiologia do envelhecimento. In: CARVALHO FILHO, E. T; PAPALÉO NETTO, M. Geriatria: fundamentos, clínica e terapêutica. São Paulo: Atheneu; 1994. p. 31-41.

MACHADO, P. A. N.; SICHIERI, R. Relação cinturaquadril e fatores de dieta em adultos. Revista de Saúde Pública, São Paulo, v. 36, n. 2, p. 198-204, 2002.

MACHADO, W. M.; FREIRE, B. F. A.; ROCHA, O. M.; AZAMBUJA, C. A. P.; OliVEIRA, M. E. C. Proposta de um questionário para caracterização da prevalência de sintomas digestivos nas doenças difusas do tecido conjuntivo. Arquivos de Gastroenterologia, São Paulo, v. 41, n. 1, p. 64-70, jan./mar. 2004.

NUTRITION SCREENING INITIATIVE. Interventions manual for professionals caring for older Americans. Washington: Nutrition Screening Initiative, 1992.

OLINTO, T. A.; NACUL, L. C.; DIAS-DA-COSTA, J. S.; GIGANTE, D. P.; MENEZES, A. M. B.; MACEDO, S. Níveis de intervenção para obesidade abdominal: prevalência e fatores associados. Cadernos de Saúde Pública, Rio de Janeiro, v. 22, n. 6, p. 1207-1215, jun. 2006.

INSTITUTO BRASILEIRO DE GEOGRAFIA E ESTATÍTICA-IBGE. Pesquisa de orçamentos familiares - 2002-2003: análise da disponibilidade domiciliar de alimentos e de estado nutricional no Brasil. Rio de Janeiro: IBGE, 2004.

PORTUGAL. Ministério da Saúde. Programa nacional contra as doenças reumáticas. Portugal: Ministério da Saúde, Direção Geral da Saúde, 2004. (Circular Normativa).
RADOMINSKI, S. C. Obesidade e doenças músculoesqueléticas. Revista Brasileira de Reumatologia, São Paulo, v. 38, n. 5, p. 275-278, set./out. 1998.

SAMPAIO, L. R. Avaliação nutricional e envelhecimento. Revista de Nutrição,Campinas, v. 17, n. 4, p. 507-514, 2004.

SAMPAIO, L. R.; FIGUEIREDO. Correlação entre o índice de massa corporal e os indicadores antropométricos de distribuição de gordura corporal em adultos e idosos. Revista de Nutrição, Campinas, v. 18, n. 1, p. 53-61, jan./ fev. 2005.

SANTOS, D. M.; SICHIERI, R. Índice de massa corporal e incadores antropométricos de adiposidade em idosos. Revista de Saúde pública, São Paulo, v. 39, n. 2, p. 163168, 2005.

SHAPIRO, D.; ANDERSON, S.; DANOFF-BURG. A pilot study of the effects of behavioral weight loss treatment on fibromyalgia symptoms. Journal of Psychosomatic Research, New York, v. 59, n. 5, p. 275282, 2005.

SOCIEDADE BRASILEIRA DE REUMATOLOGIA. Doenças reumáticas. Disponível em: <http://www. reumatologia.com.br/principais_doencas.htm $>$. Acesso em: 29 mai. 2006.

TEICHMANN, L.; OLINTO, M. T. A.; COSTA, J. S. D.; ZIEGLER, D. Fatores de risco associados ao sobrepeso e a obesidade em mulheres de São Leopoldo, RS. Revista Brasileira de Epidemiologia, São Paulo, v. 9, n. 3, p. 360-373, 2006.

YUNUS, M. B.;ARSLAN, S.;ALDAG, J. C. Relationship between body mass index and fibromyalgia features. Scandinavian Journal of Rheumatology, Denmark, v. 31, n. 1, p. 27-31, 2002.

WORLD HEALTH ORGANIZATION - WHO. Physical status: the use and interpretation of anthropometry. Geneva: World Health Organization, 1995. (WHO Technical Report Series, 854).

Obesity: preventing and managing the global epidemic. Report of a WHO Consultation on Obesity. Geneva: World Health Organization, 1997.

ZUBER, M. Criteria for diagnosis and classification of connective tissue diseases. Revista Brasileira de Reumatologia,São Paulo, v. 37, n. 1, p. 47-52, jan./fev. 1997. 
